\title{
$s$ ADMITS AN INJECTIVE METRIC
}

\author{
JOHN R. ISBELL
}

Abstract. There is an injective metric space homeomorphic with a countably infinite product of lines.

Introduction. The category of metric spaces and contractions (mappings which increase no distance) has injective objects, indeed injective envelopes [3], but little is known about their structure. Their geometry is in a sense the worst possible; for $E$ to be injective requires that however one attaches a plate to $E$ consistent with the triangle inequality, it can be contracted into $E$. Their topology is in a sense the best possible. They are topologically injective and topologically complete [2]; the locally compact ones are locally triangulable at every homotopically stable point [3]. Beyond dimension 2 [3], it is quite unknown which finite polyhedra admit injective metrics.

This note adds one example: an injective metric space $D$ is homeomorphic with the Banach space $c$ of convergent sequences, and therefore (by Kadec [4] and Anderson [1]) with a product of lines $s$, and with many other geometrically different spaces. Such an example cannot be isometric with a Banach space [2]. The homeomorphism constructed from $c$ to $D$ is not uniformly continuous, and it bends all straight lines in $c$ except for one parallel pencil. How much of that is necessary is quite unknown.

Proof. The example is the space $D$ of sequences $\left(x_{i}\right)$ of real numbers converging to a limit $\lambda$ from above, i.e. $\lambda=\inf x_{i}$, with the distance $\sup \left|x_{i}-y_{i}\right|$ induced on $D$ as a subspace of $l_{\infty} . l_{\infty}$ is injective [2].

LEMMA. The pointwise maximum of two contractions from a metric space to the real line is a contraction.

Proof. For two contractions $f, g$, and two points $x, y$, one of the four numbers $f(x), f(y), g(x), g(y)$ is largest, say $f(x)$. Then $f(x)$ $-d(x, y) \leqq f(y) \leqq(f \vee g)(y) \leqq f(x)=(f \vee g)(x)$; so $|(f \vee g)(x)-(f \vee g)(y)|$ $\leqq d(x, y)$.

THEOREM 1. $D$ is a retract of $l_{\infty}$ and therefore injective.

Proof. For $x=\left(x_{i}\right)$ in $l_{\infty}$, let $\lambda(x)=\lim \sup x_{i}$ and $p_{i}(x)=x_{i} \vee \lambda(x)$.

Received by the editors January 6, 1970.

AMS 1969 subject classifications. Primary 5435.

Key words and phrases. Metric space, injective, contraction. 
By the lemma, $p_{i}$ is a contraction. Hence $P(x)=\left(p_{i}(x)\right)$ defines a contraction, evidently a retraction upon $D$.

THEOREM 2. Dis homeomorphic with c.

Proof. Since $c$ is evidently homeomorphic with $c_{0} \times R\left(c_{0}\right.$ the space of sequences converging to 0$)$ and $D$ with $D_{0} \times R\left(D_{0}\right.$ the nonnegative sequences converging to 0 ), it suffices to give a homeomorphism $f: c_{0} \rightarrow D_{0}$. For $\left(x_{1}, x_{2}, \cdots\right) \in c_{0}$, put $x_{0}=0$. Then $f(x)=y$ is defined by $y_{i}=x_{i-1}^{-}+\sum_{j=i}^{\infty} 2^{i-j} x_{j}^{+}$(where $x^{-}=-x \vee 0, x^{+}=x \vee 0$ ). So $f$ is Lipschitzian, increasing no distance by more than a factor of 3 . By inspection, $f$ takes values in $D_{0}$.

To describe the inverse of $f$, note first that for any sequence $\left\{z_{i}: i=1,2, \cdots\right\}$, the equations $x_{0}=0$, and $2 x_{i-1}^{-}+2 x_{i}^{+}-x_{i}^{-}=z_{i}$ determine a unique sequence $\left\{x_{i}: i=0,1,2, \cdots\right\}$. If $y \in D_{0}$, define $x$ by $x_{0}=0$ and

$$
2 x_{i-1}+2 x_{i}^{+}-\overline{x_{i}}=2 y_{i}-y_{i+1}
$$

and put $g(y)=\left(x_{1}, x_{2}, \cdots\right)$.

We shall now prove the inequalities $x_{i}^{+} \leqq y_{i}$ and $x_{i}^{-} \leqq y_{i+1}$ for $y \in D_{0}$ and $i>0$. The former is immediate since either $x_{i}^{+}=0$ or from $\left(^{*}\right)$ $2 x_{i}^{+} \leqq 2 y_{i}$. To prove the latter, assume to begin with that $x_{i-1}^{-}=0$. Then $x_{i}^{-}=0$ or $x_{i}^{-}=y_{i+1}-2 y_{\imath} \leqq y_{i+1}$. Suppose now that $x_{i-1}^{-}, x_{i-2}, \cdots$, $x_{i-k}^{-}>0$, but $x_{i-k-1}^{-}=0$. Then $x_{i-1}^{+}=x_{i-2}^{+}=\cdots=x_{i-k}^{+}=0$, and a linear combination of equations $(*)$ for $i, i-1, \cdots, i-k$ gives

$$
2 x_{i}^{+}-\overline{x_{i}}=2^{k+1} y_{i-k}-y_{i+1} \text {. }
$$

Again either $x_{i}^{-}=0$ or $x_{i}^{-} \leqq y_{i+1}$.

The inequalities just proved show that $x_{i} \rightarrow 0$; i.e. $g(y) \in c_{0}$. To prove that $g$ is continuous at $y$, let $\epsilon>0$ be given. Choose $n$ so that $i \geqq n \Rightarrow y_{i}<\epsilon / 3$. If $z \in D_{0}$ and $\|z-y\|<\delta=\epsilon / 3 n$, then $\left(^{*}\right)$ shows by induction that $\left|g(z)_{i}-g(y)_{i}\right|=\left|g(z)_{i}-x_{i}\right|<3 i \delta \leqq \epsilon$ for $i=0,1, \cdots$, $n$. For $i>n$ we have

$$
\left|g(z)_{i}\right| \leqq \max \left\{z_{i}, z_{i+1}\right\}<\max \left\{y_{i}, y_{i+1}\right\}+\delta \leqq \epsilon / 3+\delta \leqq 2 \epsilon / 3
$$

and

$$
\left|g(y)_{i}\right| \leqq \max \left\{y_{i}, y_{i+1}\right\} \leqq \epsilon / 3 .
$$

Thus $\|g(z)-g(y)\|<\epsilon$.

Finally we must show that $f$ and $g$ are inverses. If $y=f(x)$ we see from the definition of $f$ that the components of $x$ satisfy $\left(^{*}\right)$. Hence $x=g(y)$; i.e., $g f$ is the identity on $c_{0}$. If $x=g(y)$ and $\bar{y}=f(x)$, then $\left(^{*}\right)$ 
and the definition of $f$ lead to $2 \bar{y}_{i}-\bar{y}_{i+1}=2 y_{i}-y_{i+1}$. Hence $\bar{y}_{i+1}-y_{i+1}$ $=2\left(\bar{y}_{i}-y_{i}\right)=2^{i}\left(\bar{y}_{1}-y_{1}\right)$. Since both $\bar{y}$ and $y$ are in $D_{0}$, this implies $\bar{y}=y$. Thus $f g$ is the identity on $D_{0}$. This completes the proof.

\section{REFERENCES}

1. R. D. Anderson, Hilbert space is homeomorphic to the countable infinite product of lines, Bull. Amer. Math. Soc. 72 (1966), 515-519. MR 32 \#298.

2. N. Aronszajn and P. Panitchpakdi, Extension of uniformly continuous transformations and hyperconvex metric spaces, Pacific J. Math. 6 (1956), 405-439; correction, ibid. 7 (1957), 1729. MR 18, 917; MR 19, 1069.

3. J. R. Isbell, Six theorems about injective metric spaces, Comment. Math. Helv. 39 (1964), 65-76. MR 32 \#31.

4. M. I. Kadec, Topological equivalence of all separable Banach spaces, Dokl. Akad. Nauk SSSR 167 (1966), 23-25 = Soviet Math. Dokl. 7 (1966), 319-322. MR 34 \#1828.

State University of New York at Buffalo, Amherst, New York 14226 